\title{
Characterization and Simulation of Hydrodynamics in the Paddle, Basket and Flow-Through Dissolution Testing Apparatuses - A Review
}

\author{
Valerio Todaro ${ }^{1}$, Tim Persoons², Geoffrey Grove ${ }^{3}$, Anne Marie Healy ${ }^{1}$, Deirdre M D’Arcy, ${ }^{4}$ \\ ${ }^{1}$ Synthesis and Solid State Pharmaceutical Centre, School of Pharmacy and Pharmaceutical Sciences, Trinity College Dublin, Dublin, Ireland \\ ${ }^{2}$ Department of Mechanical and Manufacturing Engineering, Trinity College Dublin, Dublin, Ireland \\ ${ }^{3}$ Sotax Corporation \\ ${ }^{4}$ School of Pharmacy and Pharmaceutical Sciences, Trinity College Dublin, Dublin, Ireland \\ e-mail: ddarcy@tcd.ie
}

\section{ABSTRACT}

Fluid velocity local to the dissolving surface will affect the dissolution rate. It is difficult to anticipate what local fluid velocities are present within compendial dissolution apparatuses from set flow rates or stirring rates. A range of qualitative and quantitative velocimetric techniques are available to assess and characterize hydrodynamics. These methods are frequently used in combination with computational fluid dynamics simulations to characterize and simulate hydrodynamics. Dosage form location and motion varies between apparatuses, and the presence of the dosage form itself can impact local hydrodynamics. Each apparatus described has identified hydrodynamic features which may also vary with agitation rate. This review describes the methods used to simulate and characterize hydrodynamics and summarizes some of the main findings from studies investigating hydrodynamics in the paddle, basket, and flowthrough dissolution apparatuses.

KEYWORDS: Hydrodynamics; Computational Fluid Dynamics; Dissolution Testing; Paddle Apparatus; Basket Apparatus; Flow-through Apparatus

\section{INTRODUCTION}

ydrodynamics are a commonly considered variable for modification in dissolution method development. In rotating flow apparatuses, referred to as apparatus 1 and 2 (the basket and paddle apparatus, respectively) in the USP, Ph. Eur. and Japanese Pharmacopoeias (1-3), agitation rate is represented by the revolutions per minute (rpm) of the stirrer or shaft. In the flow-through apparatus (USP/Ph. Eur. apparatus 4) $(1,2)$, agitation is represented by the flow rate, whereas in apparatus 3 , the dip rate (reciprocation rate) can be considered a representative variable of agitation. The effect of agitation rate on dissolution depends on the drug or dosage form properties, the dissolution apparatus type, and the configuration and method settings of the dissolution apparatus type. However, in general, agitation rate is expected to have a less significant effect on dissolution of a highly soluble drug, which might dissolve rapidly even in low agitation conditions, compared to a more poorly soluble drug. If a dosage form consists of, or disintegrates into, flocculent particulates, these may move with the moving fluid and, therefore, the relative fluid velocity that the dosage form is exposed to is the velocity factor relevant for drug dissolution (4). In such

* Corresponding author. a situation, agitation of the dissolution medium would be relevant to overall mixing to enable generation and retention of uniform concentration within the apparatus for reliable sampling and also for sink conditions local to the dissolving surface, if applicable. When particulates are in the small micron to nanoscale range, dissolution of a drug particle is controlled by diffusion and, therefore, agitation is again less relevant (5), aside from enabling adequate mixing. However, in many instances dissolution testing involves a dosage form which must initially undergo disintegration, and where a disintegrated mass may not be free to move with the moving fluid, such as a mass retained in a powder bed (as can happen within the basket of the basket apparatus, in the flow-through apparatus, and in the paddle apparatus if coning occurs). Therefore, for the purposes of both dissolution and generation of adequate fluid mixing in a dissolution apparatus, an understanding of hydrodynamics in the dissolution apparatuses is crucial. Agitation is induced as intended via shaft rotation, or fluid pumping rate, but could also be affected by potentially undetected variables such as vibration, shaft eccentricity, and testing unit dimensions (6); it can be affected by known adjustments such as the presence of a probe in the basket or paddle 
apparatus, or bead size or pump type in the flow-through apparatus $(7,8)$. There are many examples in the literature incorporating the effect of agitation on dissolution results and descriptions of imaging methods as applied to dosage form characterization and drug release. There are many studies specifically focusing on characterization of hydrodynamics in the compendial dissolution apparatuses. This review focuses on the methods used to characterize hydrodynamics in the dissolution apparatuses, and the main findings and characteristics of the hydrodynamics in the basket, paddle, and flowthrough apparatuses.

\section{FLOW VELOCITY}

Flow velocity relates to the linear velocity expressed as length per unit time (commonly $\mathrm{m} / \mathrm{second}$ ) of a moving fluid. Inference of the effects of flow velocity on dissolution from comparisons of flow rates or rotational agitation rates can only be made from data derived from the same apparatus. The Reynolds number $(R e)$ is a useful concept for broad comparisons of hydrodynamics in different apparatuses, as its value represents the ratio of inertial to viscous forces in the fluid motion. As such, the higher its value, the more kinetic energy is contained in rotational or turbulent motion, which is typically associated with increased mass transfer rates. The Reynolds number relates a characteristic dimension $(L)$, fluid kinematic viscosity $(v)$, and fluid velocity $(U)$ in a dimensionless term:

$$
R e=\frac{U L}{v}
$$

However, caution is still required in ensuring consistency in defining an appropriate length parameter $L$ for comparisons; for example, the dimensions of a dissolving particle could be used, or a dimension relating to the diameter of the vessel and/or shaft of the paddle apparatus.

\section{TURBULENT FLOW}

Flow can be categorized as laminar or turbulent, with a transitional region between the two flow regimes where signs of turbulence become evident within a laminar flow field. Turbulence refers to the formation, development, and interaction of rotational regions in the flow field, referred to as vortices, of varying length and velocity scales. Typically, large scale vortices are initially formed of the same order of magnitude as the vessel or stirrer. These vortices move with the flow and gradually break up into smaller eddies, passing their kinetic energy onto ever smaller scale structures in a so-called "turbulent cascade." This process is inherently stochastic and unpredictable in nature and governed by the Navier-Stokes equations of turbulent fluid motion (9). A more complex flow field which is non-unidirectional, or which is time-varying, is not necessarily turbulent flow. A laminar flow may also contain vortices which do not exhibit the same aforementioned cascade from large to small sizes as seen in fully turbulent flow. The likelihood of flow becoming turbulent is represented by the Reynolds number, as defined in Equation 1.

\section{METHODS USED TO CHARACTERIZE HYDRODYNAMICS}

\section{Methods Used to Measure Hydrodynamics}

Quantitative measures

Velocimetry covers a range of techniques which measure the motion of regions of fluid by "marking" the fluid regions, then determining the position of these marked regions at two different time points (10). The local velocity based on marker displacement, $U_{m}$, at a location, $x$, at time, $t$, is calculated using the relationship between the measured change in location, $\Delta x$, over a measured period, $\Delta t(10)$ :

$$
U_{m}(x, t)=\frac{\Delta x(x, t)}{\Delta t}
$$

The marker is usually a solid particle in a liquid but other methods can be used, such as molecules which fluoresce when activated by a laser beam or liquid droplets in gases.

\section{Particle image velocimetry and particle tracking velocimetry}

The most common quantitative velocimetry techniques include particle image velocimetry (PIV) and particle tracking velocimetry, which assume that the imaged tracer particles follow the flow perfectly. Since particle motion is typically governed by the balance of particle inertia, drag force, and gravity, the particles may exhibit a small lag in the regions of strong accelerations in the flow. This particle following behavior is characterized by its relaxation time, $\tau_{\mathrm{p}}$ :

$$
\tau_{p}=d_{p}^{2} \frac{\rho_{p}-\rho_{f}}{18_{\mu}}
$$

Where $d_{p}$ is the particle diameter, $\rho_{p}$ and $\rho_{f}$ are the particle and fluid density, respectively, and $\mu$ is the dynamic viscosity of the fluid. The Stokes number, $S t$, is a dimensionless representation of the relaxation time normalized by a characteristic time scale of the flow $\left(\tau_{f}=L / U\right)$ :

$$
S t=\frac{\tau_{p}}{\tau_{f}}=\frac{\tau_{p} U}{L}=\frac{d_{p}^{2} U}{L} \frac{\rho_{p}-\rho_{f}}{18 \mu}
$$


If the Stokes number is small $(S t<<1)$, the particles reliably follow the flow and their images can be used to infer the fluid velocity using techniques like PIV $(11,12)$.

\section{Pulsed light velocimetry}

When pulsed light velocimetry (PLV) is used, the locations of the marked fluid regions at instants in time are determined optically using pulses of light, and recorded on photographic film (10) or a digital camera sensor.

\section{Laser doppler velocimetry}

In laser doppler velocimetry (LDV), velocity measurements are taken where two laser beams cross. In the overlap region (typically a few millimeters long by a fraction of a millimeter wide), a fringe pattern is set up due to the interference of the beams. A particle crossing this measurement region will cause the light to scatter, and the varying intensity and frequency of the scattered light as the particle traverses the fringes can be measured. The frequency of the scattered light is proportional to the velocity of the particle (13).

\section{Shadowgraph imaging}

Shadowgraph imaging is a noninvasive optical technique which, in addition to quantifying the size and shape of particles at a point in time, can also quantify their absolute velocity (Figure 1) $(4,14)$. Digital images of particle-laden fluid are captured against a background of pulsed light. Individual particle velocities can be determined based on images acquired separated by a short time span. Shadowgraph imaging can also be used to observe changes in particle size over time during the dissolution process (4).

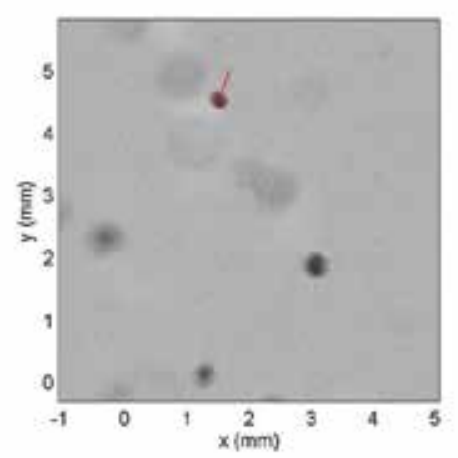

Figure 1. An example of processed shadowgraph image of dissolving particles in the USP 4 flow-through dissolution apparatus, showing size and instantaneous velocity vector of individual particles. In this case, the particle motion is near-vertical upwards.

\section{Planar laser induced fluorescence}

Planar laser induced fluorescence (PLIF) uses a light sheet to illuminate a region in a flow field. The flow should contain molecules that absorb the excitation wavelength and emit fluorescent light at a longer wavelength, such that the excitation and emitted light can be separated by means of an optical filter. Examples of such compounds include rhodamine or fluorescein. The emitted fluorescence light intensity depends on the local concentration of this compound as well as the local fluid temperature. Images thus captured in a digital camera can be calibrated using known concentrations and temperatures, resulting in a quantitative measurement of concentration or temperature fields (15).

\section{Ultrasound pulse echo}

Ultrasound pulse echo (UPE) uses a technique like PLV, where an ultrasound pulse is directed into fluid medium using a probe. The frequency at which the ultrasound pulse is generated is known. Solid particles within the fluid medium reflect the ultrasound pulse, and the frequency at which the returning ultrasound beam is detected by the probe is an indication of how fast the particle (and, hence, the fluid) is moving. In other words, it is the frequency shift of the ultrasound pulse which enables determination of fluid velocity (16).

\section{Magnetic resonance imaging}

Magnetic resonance imaging (MRI) flow techniques use a magnetic field gradient and radio frequency pulses to produce spin echoes from the $1 \mathrm{H}$ nuclei in water molecules. Using a pulse sequence technique such as pulsed gradient spin echo, the magnitude of the echo signal generated can be related to the motion of the water molecules, with a larger echo representing a slower moving population of molecules. By acquiring multiple readings per second, a visualization of the flow can be constructed. Use of both NMR and MRI-based methods to investigate the hydration, physical changes, and drug release from drug delivery systems in the USP 4 flowthrough apparatus have been described (17-19).

\section{Qualitative measures: flow visualization}

using dye studies

Flow visualization can cover any technique which enables the fluid flow to be visualized, and allows for the qualitative assessment of hydrodynamics and mixing patterns of a flow field. A common method involves injecting a dilute dye into the fluid medium and monitoring (visualizing) its motion and, thus, the hydrodynamics from the point of injection into the fluid domain. The density and viscosity of the dye solution should be similar to the fluid being investigated. Physicochemical characteristics of the fluid medium, such as $\mathrm{pH}$, can also be utilized to visualize fluid flow patterns. The hydrodynamics around a dissolving 
salicylic acid tablet have been visualized by incorporating a phenolphthalein indicator in a dissolution medium of dilute sodium hydroxide in the paddle and basket dissolution apparatuses at $0,25,50$, and $100 \mathrm{rpm}$. As the surface of the tablet dissolved, the pink color of the phenolphthalein indicator in the clear sodium hydroxide dissolution medium indicated the flow direction in the medium surrounding the tablet (20). Laser-induced fluorescence, introduced as a quantitative technique in the previous section, can also be used to visualize and characterize mixing in a flow field (21). Flow visualization can be achieved by the photography of the flow field capturing the fluorescence emission (22).

\section{METHODS USED TO SIMULATE HYDRODYNAMICS}

The physics of fluid flow are governed by the principles of conservation of mass, momentum, and energy. These can be represented mathematically by the NavierStokes partial differential equations, or by their integral formulations. The process of numerically solving these equations in space and/or time is called computational fluid dynamics (CFD). Different solving approaches can be used based on the finite difference method (FDM), finite element method (FEM) or finite volume method (FVM). FDM is typically not used except for the simplest geometries and flow conditions. FEM can be used for laminar or generally "well-behaved" flows. For more complex flows involving turbulence or compressibility, FVM is used, in which case the governing equations are solved in their conserved or integral form (23). For dissolution studies, typical flow conditions are such that either FEM or FVM can be used. In the field of pharmaceutics, the hydrodynamic conditions present in dissolution studies are of interest in terms of their effect on dissolution rates, variability in dissolution test results, and relevance to in vivo hydrodynamic conditions.

In a typical CFD experiment, a simplified geometry of the flow field of interest is often created. In a symmetrical problem, a two-dimensional (2D) geometry can suffice, and requires reduced computational resources as compared to three-dimensional (3D) geometry. A 3D geometry is typically used to model nonsymmetrical geometries, or if greater detail from the simulation is required (e.g., more complex flow is anticipated, noting that a symmetrical geometry does not necessarily imply a symmetrical flow field will develop). The created geometry is divided into discrete elements or cells, with the number and size of elements generated affecting the accuracy of the simulated flow field. Initial boundary conditions are defined (e.g., rotating agitation rate in the paddle apparatus or inlet inflow rate in the flowthrough apparatus) along with fluid properties. The equations of momentum and mass conservation (for each species) are solved at each defined point in the flow field. The energy equation can be omitted in the case of isothermal flows, as is the case in dissolution testing. As the hydrodynamic properties at each point in the flow field must be contingent with the values at neighboring points, the equations cannot be solved in isolation for each point. Thus, to arrive at an acceptable solution, the problem requires repetitive numerical iteration rather than a direct analytical mathematical solution. For time-varying flow fields, a new flow field solution is generated for each time point, with the solution at the preceding time point providing the initial conditions. In cases of turbulent flow, the basic fluid motion and mass conservation equations are not adequate alone and must be supplemented with models which aim to simulate the generation of turbulent characteristics in the flow field. A review of such approaches and models is beyond the scope of this review, however, the addition of extra models (e.g., turbulence models), while adding to the accuracy of the solution, also adds to the complexity of the problem being solved and requires significant computational resources. Furthermore, increasing the density of the domain mesh (the density of points in the problem flow field), employing a 3D (rather than 2D) geometry, and generating a time-dependent simulation can all add significantly to the computational resources required. It is therefore crucial to understand from the outset the aim of a CFD model and what the intended accuracy of the simulation is with relation to features of the flow field being investigated. Some form of validation of a CFD solution is usually presented, which will give an indication of solution accuracy for an intended purpose. Validation methods include the qualitative and quantitative hydrodynamic methods outlined above.

\section{HYDRODYNAMIC CHARACTERISTICS OF DISSOLUTION APPARATUSES}

\section{Paddle Dissolution Apparatus}

The harmonized (1-3) paddle apparatus (Apparatus 2) is the most widely used dissolution apparatus for the evaluation of dissolution profiles of solid pharmaceutical dosage forms. Using velocimetric techniques and CFD, it is possible to experimentally map and computationally predict the velocity distribution inside the apparatus.

The following section presents a general overview of the hydrodynamic characteristics of the paddle dissolution apparatus using findings from a combination of studies using both velocimetric measurements and 
CFD simulations. Experimental and simulated results were generated under a range of conditions, some at room temperature and some at $37^{\circ} \mathrm{C}$. The effect of these temperatures on hydrodynamics would most notably affect the fluid viscosity value (viscosity of water is $1 \mathrm{cp}$ at approximately $20^{\circ} \mathrm{C}$ and approximately $0.74 \mathrm{cp}$ at $37^{\circ} \mathrm{C}$ ). This difference in viscosity could affect some local velocity values, but would not be expected to impact the overall nature of the observed hydrodynamic features of the flow field in the vessel.

\section{General flow features}

The predominant component of velocity throughout the vessel is the tangential component, with axial and radial components varying according to recirculation loops, and an increased radial component in the region of the paddle impeller due to radial pumping. Recirculation loops have been identified in both the upper and lower sections of the vessel, with a weak positive axial flow under the impeller in the center of lower part of the vessel. Overall fluid velocity values and shear rates in the vessel base are very low, especially at the center of the vessel base compared to other regions of the vessel $(13,21,24-27)$.

These recirculation loops could suggest segregated flow regions between the upper and lower regions of the vessel. However, dye studies and particle tracer simulations demonstrated segregated zones are not present, and mixing within the vessel is not likely to be problematic $(21,28)$ from the perspective of sampling (upper vessel) relative to the location of the dissolution process (lower vessel), aside from extremely early sampling times (28).

Contours of CFD-simulated velocity values on a vertical plane through the vessel at $50 \mathrm{rpm}$ are shown in Figure 2.

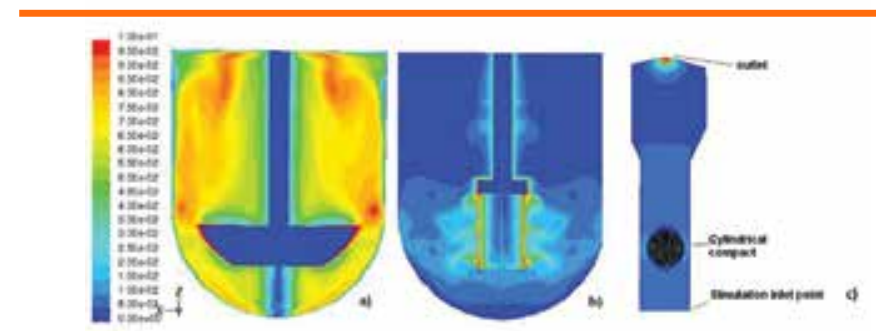

Figure 2. Contours of velocity magnitude ( $\mathrm{m} / \mathrm{second}$ ) from CFD simulations of a) the paddle apparatus at $50 \mathrm{rpm} ; \mathrm{b}$ ) the basket apparatus at $100 \mathrm{rpm}$; and c) $12 \mathrm{~mm}$ flow-through cell, $17 \mathrm{~mL} / \mathrm{min}$, at 0.15 seconds of pulse (just after peak inflow). All velocities above $0.1 \mathrm{~m} / \mathrm{second}$ are shown in red. In-house images generated using methods detailed in previous studies. ${ }^{a, b, c}$

acCarthy, et al. AAPS PharmSciTech. 2003, 4 (2), 83-98 (24).

${ }^{b} D^{\prime}$ Arcy et al. Eur. J. Pharm. Sci. 2006, 27 (2-3), 259-67 (44).

'D'Arcy et al. Pharm. Res. 2010, 27 (2), 246-58 (53).

Abbreviations: CFD, computational fluid dynamics.
Hydrodynamics in the lower hemispherical

section of the vessel

Under the standard operating conditions mandated by the dissolution test procedure, with no tablets present in the vessel, the shear strain environment in USP Apparatus 2 is highly heterogeneous with strain rate and fluid velocities varying significantly along the vessel base $(21,24-27,29)$. These heterogeneous hydrodynamic conditions are present in the vessel base region where a dosage form is likely to be located. Dissolution results from both disintegrating and non-disintegrating dosage forms have varied with location in the vessel base, with slower dissolution observed from dosage forms located in the center in comparison to those located off-center $(21,25,30,31)$. These differences in dissolution rates were related to local fluid velocity and strain rate variations in the vessel base $(21,25,30,31)$. Fixing the position of nondisintegrating dissolving compacts either centrally or offcenter was noted to result in lower variability in dissolution rate than that observed from the compacts not affixed to any position (30).

\section{Effect of rotation speed}

A proportional increase in axial and tangential velocities with rotation speed has been reported from CFD, UPE, and LDV studies $(16,27,28)$. Studies using CFD, PLIF, and LDV have also demonstrated that changes in agitator speed from 50 to $100 \mathrm{rpm}$ resulted in different fluid velocities but similar simulated flow patterns $(21,27)$. The strain rate magnitude increased with increased rpm, but the distribution of variation of strain rate in the apparatus was similar at 50 and $100 \mathrm{rpm}$, thus, the homogeneity of the shear in the apparatus was not improved with increasing agitation rate (21). Very low velocities are retained in the central core region below the impeller with increasing rpm, however, the tangential velocity component increases with rpm, and, outside of the central core region, axial and radial velocities also increase. In this region, axial velocity tended to increase with increasing rpm whereas observations suggest radial velocities decreased in some locations near the vessel base with an increasing rpm (27). Furthermore, the central region at the vessel base of low shear strain decreases with increasing rpm (27).

\section{Coning}

The agitation in the USP Apparatus 2 can generate a cone of disintegrated/particulate material at the bottom of the vessel, underneath the paddle, limiting the dissolution of the pharmaceutical dosage form in this region. The recirculation loop described in the lower part of the 
vessel may not penetrate the very low velocity region at the center of the vessel base (26), which explains this frequently observed coning phenomenon. It is possible to predict the minimum rotation speed at which the cone will disappear based on an application of the Zwietering equation $(32,33)$. The decreased propensity towards coning with increased rpm is supported by the observation that increased tangential velocities with increased agitation rates will result in increased centrifugal force pulling particles/tablet fragments from the inner core at the vessel base outwards, and promote particle suspension in the dissolution medium (27). However, centrifugal force acting on a particle will be influenced by the density difference between the particle and the liquid. Furthermore, as illustrated by Higuchi et al. (2014) (32) on the application of the Zwietering equation to predict the minimum speed required to prevent coning in the paddle apparatus, an increased particle size and density will require an increase in velocity to prevent coning for the same liquid. Therefore, for any disintegrating tablet, the size and location of the disintegrated mass will influence the local velocities to which particles are exposed, and the size and density of those particles will influence whether they will remain as a cone in the center. As tangential velocities increase with increasing distance from the center of the vessel, even within the inner core, and as CFD simulations presented by Bai et al. in the lower region of the vessel accurately represent the tangential velocities present as validated by LDV measurements, these CFD simulations do substantiate the concept that increasing velocity from 50 to $75 \mathrm{rpm}$ will decrease the likelihood of coning (27). However, as coning also depends on tablet and particle properties, it cannot be said that such an increase will eliminate the likelihood of coning completely. An interesting and thorough critique on the effect of agitation on the propensity for cone formation is given in Bai et al. 2011 (27), and the reader is referred to this for further insight into this topic.

Along with the numerous studies in which the main features of and dissolution conditions in the paddle apparatus that directly influence the fluid dynamics of the system have been described through CFD and experimental velocimetry, some possible adjustments to reduce the variability in dissolution results have also been suggested. Furthermore, variations in dissolution test set up (e.g., the presence of a sampling probe or use of a mini-vessel) can affect hydrodynamics. The hydrodynamic implications of some of these variations and adjustments to the dissolution set up are outlined in the following section.
Impeller positioning and vessel shape

The impeller position is an important factor impacting hydrodynamics. The standard central position of the impeller generates a symmetrical distribution of axial and radial components of velocity with respect to the center line of the vessel, with the formation of a slow fluid velocity zone under the center of the paddle.

CFD simulations have indicated increasing the clearance between the impeller and vessel base led to better mixing in the vessel overall, however, this also resulted in an increase in the size of the low velocity region in the lower hemispherical section of the vessel $(34,35)$. In contrast, when the paddle is placed very close to the vessel base, a breaking flow is produced in this area along with a reduced or eliminated lower recirculation zone (34).

Interestingly, when the impeller is situated $2 \mathrm{~mm}$ offcenter, CFD simulations suggest asymmetrical flow features are present, which move as the paddle rotates, resulting in the removal of the poorly mixed region in the center of the vessel base. The strain rate is more evenly distributed along the wall of the lower hemispherical portion of the vessel, promoting better mixing (29). Such a deliberate removal of symmetry was also presented by Wang and Armenante (2012) with the impeller positioned $8 \mathrm{~mm}$ off-center, resulting in a more robust dissolution testing environment through the elimination of the poorly mixed zone at the center of the vessel base (36). The effect of tilting the vessel relative to the impeller was presented by Mirza et al. (37). Mirza et al. hypothesized that tilting the vessel from the central position by $3.0 \mathrm{~mm}$ and $4.5 \mathrm{~mm}$ resulted in the disruption of the cone due to the increased flow at the bottom of the vessel. Tilting resulted in dissolution release rates increasing in a manner similar to that resulting from an increase in agitation rate.

The shape of the vessel base is another important factor affecting distribution of shear rates in the vessel and the tendency for coning to occur at the center of the hemispherical vessel base. Using a vessel with a flat bottom did not result in increased performance over the standard vessel (37), supporting observations by Ameur et al. (34) that a dish-bottomed vessel results in better mixing as compared to a flat-bottomed vessel, and the hydrodynamics in a vessel with a hemispherical base result in a greater well-stirred region.

The peak vessel incorporates a raised convex section at the center of the hemispherical vessel base intended to prevent the dosage form from settling in a low-velocity zone. Therefore, a dosage form in the peak vessel should only be exposed to the higher shear region outside of 
the center of the vessel base. The use of a peak vessel increased dissolution from both low and high-solubility drugs (both known to be cone-forming) (37). Whereas the local variation in shear rate is reduced in the peak vessel, higher shear rates might result in a loss of discriminative ability of the dissolution test (35). However, with respect to the peak vessel being used to avoid the problem of coning, the more homogenous shear rates in the peak vessel environment are advantageous in promoting more reproducible dissolution results while avoiding artificially low or suppressed dissolution resulting from cone formation.

The impeller design also influences the hydrodynamics and shear rate distribution. It was observed in CFD simulations that increasing the lower blade edge promotes increased heterogeneity of shear forces near the vessel base. Additionally, when the ratio between the diameter of blades and the diameter of tank is small, the shear rates observed in the base of the vessel are weak. However, when the ratio is too high, the formation of a larger dead zone at the center of the vessel base has been reported (34).

Using a Rushton or axial pitched-blade impeller, lower shear rates were noted overall compared with the paddle apparatus; however, the modified impeller blades were smaller. In the lower region of the vessel, shear rate increase was magnified with the Rushton impeller compared to the standard paddle configuration, and more severely heterogeneous shear was noted with the pitched blade turbine at an equivalent Reynolds number. There is, therefore, no reported advantage over the standard configuration with the paddle using these alternate impeller types (35).

\section{Presence of probe}

Zhang et al. demonstrated the effects of the introduction of an optic probe to the paddle apparatus (38). Tablet dissolution generally increased when the probe is present, which is likely due to two effects. The presence of the probe is like a baffle effect in a symmetric system like the USP vessel and is suggested to disrupt the tangential flow while increasing the radial and axial flow components. The second effect relates to the introduction of a small object (the probe) inside the symmetrical agitation system, resulting in a hydrodynamically asymmetrical system. Further studies are required to elucidate and quantify the hydrodynamic effects of probe presence, positioning, and type.

\section{Mini-vessels}

Different models of small-volume paddle and vessel apparatus are commercially available as an alternative to the standard paddle apparatus to perform dissolution experiments using lower volumes of media. Especially for low-dose formulations, the utilization of the minivessel apparatus can improve the analytical evaluation of dissolution by using a smaller volume of test media. Moreover, it can generate a significant cost reduction, particularly when using biorelevant media (39-41). However, there is no standard size of mini-vessel set-up, and several configurations are reported in the literature.

Klein and colleagues $(2006,2008)$ investigated dissolution at different agitation rates in an Erweka mini-paddle apparatus with $250 \mathrm{~mL}$ of dissolution medium and a standard paddle apparatus with $500 \mathrm{~mL}$ of dissolution medium $(39,40)$. Dissolution rates were similar from both apparatuses when the agitation rate was $75 \mathrm{rpm}$ in the standard apparatus and 75-100 rpm in the minivessel. This may be due to similar relative velocities being experienced by dissolving particles once coning did not occur rather than proof that the hydrodynamic environment within the apparatuses were similar under these conditions. Scheubel et al. compared dissolution using $150 \mathrm{~mL}$ dissolution medium in a Sotax small volumevessel to the standard paddle apparatus (using $500 \mathrm{~mL}$ or $900 \mathrm{~mL}$ dissolution medium), for a range of dosage forms (41). The agitation rate required for similar dissolution from each apparatus was dependent on the drug release mechanism. For example, in several cases, an agitation rate of $125 \mathrm{rpm}$ in the mini-vessel resulted in similar dissolution to $50 \mathrm{rpm}$ in the standard paddle apparatus; however, for a slow-release formulation, there was similar drug release at $50 \mathrm{rpm}$ in both apparatuses.

Stamatopolous et al. used PLIF and PIV to explore hydrodynamics in an Antech mini-paddle dissolution apparatus, using media of different viscosities, along with dissolution testing (42). PIV results revealed two recirculation zones. A low velocity region was noted under the impeller, and observed to increase in size with increasing viscosity. In the upper vessel, increasing viscosity reduced recirculation patterns, and, in simple buffer solution, the position of the sampler created another circulation loop which was not evident in more viscous solutions. The PLIF technique showed that, in viscous media, a segregated zone is characterized by a high amount of dye, especially in the bottom of the vessel due to high inhomogeneous mixing. Some delay in mixing between the upper and lower zones resulted in differences in dissolution profiles depending on sampling location. 
Locher et al. reported the effect of agitation rate on partitioning into the organic phase in a mini-scale biphasic dissolution model with two impellers: one in the aqueous phase and one in the organic phase. In their study, the paddle rotation speed in the aqueous phase had a greater effect than that in the organic phase when the dual paddle was used (43).

Overall, due to the non-standardized and relatively novel nature of the mini-vessel concept, there is little known specifically about hydrodynamics within these apparatuses. The dissolution set-up is not always presented in terms of Reynolds numbers, which could be a useful basis on which to consider investigating the interchangeability of dissolution tests between the standard set-up with a mini-vessel for specific formulations. In addition to Reynolds numbers, attention must be given to coning, mixing, and sampling in these apparatuses in order to generate reliably comparable dissolution profiles between mini-vessels and standard apparatuses.

\section{BASKET DISSOLUTION APPARATUS}

The harmonized (1-3) basket apparatus (Apparatus 1) is the second most widely used dissolution apparatus, allowing users to perform dissolution tests on floating pharmaceutical formulations without the need to use sinkers. The basket apparatus uses the same instrument and vessel type as the paddle apparatus, typically with a vessel volume capacity of $1 \mathrm{~L}$, however, in place of the paddle, a cylindrical basket with mesh openings holds the dosage form. Inadequacy of mixing at lower rpm, clogging of the mesh, and the "sieving effect" by the mesh of particles of different sizes are possible causes of variation between dissolution results. The hydrodynamics involved in the dissolution process using the basket apparatus have been investigated using CFD (44) and UPE (16). Comparing the velocities obtained using UPE with the results recorded using CFD, higher velocity values were detected by UPE (44). This is most likely due to the CFD-simulated flowfield being a time-averaged solution and the UPE method capturing maximum values at a particular location on each plane of the vessel. The UPE measurements were not taken within or near the basket itself, where the highest velocities are generated, and differences between simulated and measured velocities at the planes investigated were less than $10 \%$ of the maximum simulated velocities in the basket apparatus (44). Nonetheless, within the observed flow field, some time-dependent features were present resulting in increased velocities being observed compared to the CFD simulation (44).

\section{Hydrodynamic features}

Contours of CFD-simulated velocity values on a vertical plane through the vessel at $100 \mathrm{rpm}$ are shown in Figure 2. Hydrodynamics in the basket dissolution apparatus using CFD simulations and UPE have been described in detail $(16,44,45)$.

An ascending axial flow was noted under the basket in CFD simulations with the fluid entering axially at the base of basket and exiting radially at the sides near the basket base. Furthermore, positive and negative radial and axial flows were simulated within the basket, along with regions of higher axial and radial velocity just outside the basket (44).

As the basket itself acts as the stirrer, the highest velocities are in the region of the basket sides, as suggested in CFD simulations, and are likely to vary in a time-dependent manner. Other areas in the basket apparatus are characterized by low velocity, particularly in the center of the basket and in the upper and lower (i.e., base) regions of the vessel. These differences in hydrodynamics within the basket and at the base of the vessel are particularly notable when considering the effect of formulation on dissolution, as particles which are retained within the basket will likely experience different hydrodynamic conditions to those which would descend to the vessel base (Figure 2) (44).

Comparing the CFD simulated hydrodynamics from USP (basket) Apparatus 1 with USP (paddle) Apparatus 2, the maximum simulated absolute velocity within the basket at $50 \mathrm{rpm}$ is only slightly higher than the maximum simulated velocity at $1 \mathrm{~mm}$ from the top of a compact located at the center of the base of the vessel in the paddle apparatus operating at $50 \mathrm{rpm}$ (Tables 1 and 2) (30, 44, 45). An estimate of the relative velocity a tablet rotating in the basket of the apparatus might experience would suggest the relative velocity at $100 \mathrm{rpm}$ in the basket apparatus (45) is closer to that at $50 \mathrm{rpm}$ at the center of the vessel base in the paddle apparatus (Tables 1 and 2) $(30,45)$. However, consideration must be given to the exposure of the lower surface of the tablet in the basket apparatus and the contribution of natural convection, particularly from a downward facing surface in the relatively low-velocity environment of the bottom of the basket. Therefore, comparable dissolution results have been observed from a non-disintegrating tablet within the basket of the basket apparatus and at the base of the vessel in the paddle apparatus at the same rpm $(44,46)$. These findings illustrate how hydrodynamic data, such as that presented in Figure 2 , cannot be used in isolation to predict dissolution rates in different apparatuses. 
Table 1. Velocity Values in Different Conditions of the Basket Dissolution Apparatus $^{a, b}$

\begin{tabular}{|r|c|c|c|}
\hline Apparatus & $\begin{array}{c}\text { Basket } \\
\text { Apparatus }\end{array}$ & $\begin{array}{c}\text { Basket } \\
\text { Apparatus }\end{array}$ & $\begin{array}{c}\text { Basket } \\
\text { Apparatus }\end{array}$ \\
\hline Agitation rate & $50 \mathrm{rpm}$ & $50 \mathrm{rpm}$ & $100 \mathrm{rpm}$ \\
\hline $\begin{array}{r}\text { Location and } \\
\text { type of velocity } \\
\text { data }\end{array}$ & $\begin{array}{c}\text { Relative velocity } \\
\text { magnitude in } \\
\text { basket }\end{array}$ & $\begin{array}{c}\text { Maximum } \\
\text { absolute } \\
\text { velocity } \\
\text { magnitude in } \\
\text { basket }\end{array}$ & $\begin{array}{c}\text { Relative velocity } \\
\text { magnitude in } \\
\text { basket }\end{array}$ \\
\hline $\begin{array}{r}\text { Velocity } \\
(\mathrm{m} / \text { second) }\end{array}$ & 0.026 & 0.057 & 0.059 \\
\hline
\end{tabular}

${ }^{a} D^{\prime}$ Arcy et al. Eur. J. Pharm. Sci. 2006, 27 (2-3), 259-67 (44).

${ }^{b} D^{\prime}$ Arcy, D. M. Use of computational fluid dynamics to investigate the relationship between hydrodynamics and rates of dissolution; Dublin: Trinity College Dublin, 2007 (45).

Table 2. Velocity Values Near Tablets Located Centrally and Off-Center at the Base of the Paddle Dissolution Apparatus ${ }^{a, b}$

\begin{tabular}{|r|c|c|c|c|}
\hline Apparatus & $\begin{array}{c}\text { Paddle } \\
\text { apparatus } \\
\text { (compact in } \\
\text { central position) }\end{array}$ & $\begin{array}{c}\text { Paddle } \\
\text { apparatus } \\
\text { (compact in } \\
\text { central position) }\end{array}$ & $\begin{array}{c}\text { Paddle } \\
\text { apparatus } \\
\text { (compact } \\
\text { off-center - } \\
\text { position 1) }\end{array}$ & $\begin{array}{c}\text { Paddle } \\
\text { apparatus } \\
\text { (compact } \\
\text { off-center - } \\
\text { position 2) }\end{array}$ \\
\hline $\begin{array}{r}\text { Agitation } \\
\text { rate }\end{array}$ & $50 \mathrm{rpm}$ & $50 \mathrm{rpm}$ & $50 \mathrm{rpm}$ & $50 \mathrm{rpm}$ \\
\hline $\begin{array}{r}\text { Location } \\
\text { and type of } \\
\text { velocity data }\end{array}$ & $\begin{array}{c}\text { Maximum } \\
\text { velocity } \\
\text { magnitude, } \\
\text { top surface } \\
\text { of compact }\end{array}$ & $\begin{array}{c}\text { Maximum } \\
\text { velocity } \\
\text { magnitude, } \\
1 \text { mm from } \\
\text { side surface } \\
\text { of compact }\end{array}$ & $\begin{array}{c}\text { Maximum } \\
\text { velocity } \\
\text { magnitude, } \\
1 \text { mm from } \\
\text { top surface } \\
\text { of compact }\end{array}$ & $\begin{array}{c}\text { Maximum } \\
\text { velocity } \\
\text { magnitude, } \\
1 \text { mm from } \\
\text { top surface } \\
\text { of compact }\end{array}$ \\
\hline $\begin{array}{r}\text { Velocity } \\
\text { (m/second) }\end{array}$ & 0.049 & 0.067 & 0.085 & 0.090 \\
\hline
\end{tabular}

${ }^{a} D^{\prime}$ Arcy et al. J. Pharm. Pharmacol. 2005, 57, 1243-50 (30).

${ }^{b} \mathrm{D}$ 'Arcy, D. M. Use of computational fluid dynamics to investigate the relationship between hydrodynamics and rates of dissolution; Dublin: Trinity College Dublin, 2007 (45).

\section{FLOW-THROUGH DISSOLUTION APPARATUS}

The Flow-Through Cell (FTC) apparatus (USP Apparatus 4) method was developed in 1957 by the US Food and Drug Administration and introduced into the USP, Ph. Eur. and Japanese Pharmacopoeia as an official apparatus to study the dissolution profile of pharmaceutical solid dosage forms (47). The FTC reportedly has ideal hydrodynamic conditions for homogenous, mild agitation (48). It is of interest to explore this assertion and characterize the hydrodynamics within the apparatus, and studies have been conducted using CFD and velocimetric methods. The following section provides an overview of hydrodynamics and dissolution in the FTC apparatus, while a comprehensive account of the effect of the pulsing pump on hydrodynamics in the FTC apparatus has been previously presented (49).
In contrast to other dissolution apparatuses, the FTC apparatus uses a piston pump that generates a sinusoidal or semi-sinusoidal flow profile. This results in a pulsing flow resulting in variation in the fluid velocity with time and, thus, an inconstant Re. While a constant inflow can also be used, the current section focuses on the hydrodynamics in the pulsing flow field. In the FTC apparatus, it is possible to use two different sizes of cell, having internal diameters of $\varnothing 22.6 \mathrm{~mm}$ or $\varnothing 12 \mathrm{~mm}$ and providing volumes of approximately $19 \mathrm{~mL}$ and $8 \mathrm{~mL}$, respectively (47). The larger $\varnothing 22.6 \mathrm{~mm}$ cell has lower average linear fluid velocities than the smaller cell at equivalent flow rates due to an increased cross-sectional area. Dissolution in the FTC apparatus can be influenced by several factors such as the flow rate, cell temperature, tablet orientation, level of deaeration, and size of the beads used in the cell $(7,50)$. Moreover, during the pulsing flow, it is possible to distinguish an initial inflow phase $(0-0.25 \mathrm{~s})$ characterized by the pump discharge, followed by a suction phase $(0.25-$ $0.5 \mathrm{~s})$ where the inflow velocity is zero (49). The pulse frequency is $120 \pm 10$ pulses per minute regardless of the selected flow rate, with standard flow rates of 4,8 , and $16 \mathrm{~mL} / \mathrm{min}(1)$.

A rigorous analysis of flow regimes in the flow-through cell was presented by Kakhi (51). Kakhi also used CFD to compare the hydrodynamics in the $\varnothing 22.6 \mathrm{~mm}$ cell, both with and without the inclusion of glass beads $(\varnothing 1 \mathrm{~mm})(52)$. During the latter half of the inflow phase, the flow field appears asymmetric and non-uniform in the proximity of the ruby bead in the simulation with no glass beads. However, there is a flat, symmetrical velocity profile over a notable portion of the dissolution test section (52). Moreover, when the ruby bead is included and the lower conical section of the column is packed with the glass beads, no notable difference is predicted between open and packed column configurations for the shear stress distributions in terms of trend and magnitudes in the test region of the cell. The fluid dynamics around a tablet in the tablet holder would, therefore, be expected to be essentially similar in the $\varnothing 22.6 \mathrm{~mm}$ cell using the "packed column" and "open column" configurations (i.e., with and without glass beads, respectively), at a flow rate of $16 \mathrm{~mL} / \mathrm{min}(51,52)$. Another notable feature observed from the simulations was the wall shear stresses on the tablet surface varied markedly during the acceleration and deceleration phases of the pump discharge, at time points when the inflow velocity would be the same. This feature was attributed to flow reversal predicted in the boundary layer surrounding the tablet, likely due to the fluid moving against an adverse pressure gradient because of the pulsating nature of the flow (52). 
The presence of some flow reversal and boundary layer detachment during the suction phase was simulated using CFD in both the larger $(\varnothing 22.6 \mathrm{~mm})$ and smaller ( $\varnothing 12 \mathrm{~mm}$ ) cell (53). These CFD simulations demonstrated that significant variations in hydrodynamics occur over the course of the pump pulse, with the flow rate also impacting on the residual fluid motion present during the suction phase.

The effect of the pulsing flow on the dynamics of dissolution have also been simulated using CFD (53), with simulations illustrating the diffusion boundary layer thickness changing during the pump pulse in the $\varnothing 12 \mathrm{~mm}$ cell, suggesting an impact on the local dissolution kinetics. The boundary layer thickness is greater during the zeroinflow velocity period than the high inflow velocity period, particularly at the upper and lower edges of the vertical plane of a vertically oriented compact (53). Furthermore, the dissolution rate from a non-disintegrating tablet in the larger $\phi 22.6 \mathrm{~mm}$ cell at $8 \mathrm{~mL} / \mathrm{min}$ was found to be lower than that in a system with no forced convection (i.e., a free convection system), suggesting that at very low flow rates in the FTC apparatus, the flow regime can inhibit dissolution (53).

In addition to this noted inhibitory effect of the flow regime on dissolution at low flow rates, the solubility of the dissolving solute was observed to be a further confounding factor (54). The reduction in dissolution rate in the FTC apparatus, in comparison with the free convection system, was more marked during the dissolution of a lactose compact than a benzoic acid compact in $0.1 \mathrm{M} \mathrm{HCl}$ (54). The higher solubility of lactose results in a denser saturated solution at the dissolving surface. Simulations of the hydrodynamics in the presence of this denser solution at the surface suggested a notable gravityinduced downward flow from this denser solution, and a marked difference in the hydrodynamics in the FTC in the presence of a saturated solution of (more soluble) lactose at the dissolving surface compared to a saturated solution of (less soluble) benzoic acid (54). Thus, the interaction between the upward pulsing flow and the downward flow due to both flow reversal and natural convection from solute solutions will be dependent on the flow rate and density of the solute solution (54). The effect (simulated and observed) of density gradients on hydrodynamics and dissolution in constant flow, very low velocity flowthrough environments was also presented by Stevens and Missel (55).

In terms of particulate systems in the FTC apparatus, shadowgraph imaging has demonstrated that suspended particles do follow a somewhat pulsing motion, however, the motion of the particles can be complex (4). In a hypothetical situation, a change in flow rate should not impact the relative fluid velocity, however, the effect of flow rate on dispersion of the powder bed and particle interactions with the cell surfaces will be affected by flow rate. Shadowgraph imaging has proven useful for exploring particle behavior within the FTC apparatus in terms of particle velocities, sizes, and particle agglomeration tendencies $(4,14)$.

MRI has been used to visualize and quantify flow in the FTC apparatus, both with and without a cylindrical disk "tablet" (representing the dosage form) in the cell (56). A very detailed description of observed hydrodynamics is provided by Shiko et al. (56). In the $\varnothing 12 \mathrm{~mm}$ cell, the flow profile was relatively steady, aside from the motion induced by the pulsation of the pump, at 4 and $8 \mathrm{~mL} / \mathrm{min}$. In contrast, the flow field at $16 \mathrm{~mL} / \mathrm{min}$ exhibited much greater heterogeneity. In the $\varnothing 22.6 \mathrm{~mm}$ cell at 8 and 16 $\mathrm{mL} / \mathrm{min}$, flow was characterized by a central jetty and peripheral recirculation patterns. Fluid velocities around a tablet oriented both horizontally and vertically in the $\varnothing 12 \mathrm{~mm}$ cell at $8 \mathrm{~mL} / \mathrm{min}$ were also described. The horizontally oriented tablet experienced increased axial and radial fluid velocities due to the reduced crosssectional area available for flow, and radial velocity distributions differed over the course of the pulse, depending on tablet orientation, with greater variation around the vertically oriented tablet (56). The effect of variation in hydrodynamics relative to tablet orientation was previously presented in terms of observed effects on dissolution. Horizontally oriented non-disintegrating tablets exhibited higher dissolution rates than vertically oriented tablets (57). Whereas in that study, the horizontal tablets were not placed directly on the glass beads, the cross-sectional area is nonetheless reduced in comparison to a vertically oriented tablet. MRI has additionally been used to visualize the dissolution process of tablets through visualization of water movement around and into the dosage form, suggesting a potential future application of this technology to characterize the dissolution process (58).

The pump pulse can operate following a semi-sine wave or a full-sine wave profile. Yoshida et al. reported dissolution and hydrodynamic data using PIV in the FTC apparatus using pumps delivering the medium as a constant flow, or pulsing flow following a semi-sine wave or full-sine wave profile $(8,59)$. PIV results from the $\varnothing 12 \mathrm{~mm}$ cell illustrated the higher instantaneous flow rate from the semi-sine wave pump during the discharge phase of the pump, with a slow decrease during the suction phase 
and a larger increase in flow rate towards the end of the cycle from the full-sine wave pump (8). Yoshida et al. also observed a slightly faster dissolution of the salicylic acid standard tablets in the $\varnothing 12 \mathrm{~mm}$ cell using the semi-sine wave pulse in comparison to dissolution using the pump which delivered a full-sine wave profile, possibly due to the higher maximum instantaneous flow that is provided by the semi-sine wave pump during the discharge phase, reducing the diffusion boundary layer thickness. Furthermore, the effect of flow rate on dissolution was evident in the $\varnothing 12 \mathrm{~mm}$ cell but not so clear in the $\emptyset 22.6 \mathrm{~mm}$ cell (8). A lack of effect of flow rate on the dissolution of salicylic acid reference tablets in the $\varnothing 22.6$ $\mathrm{mm}$ cell has been observed previously (57).

Using disintegrating prednisone reference tablets, the flow rate affected the dissolution profile in both the $\varnothing 12 \mathrm{~mm}$ and $\varnothing 22.6 \mathrm{~mm}$ cells, and the pump type (sinusoidal vs semi-sinusoidal) had a notable effect on dissolution in the $\varnothing 22.6 \mathrm{~mm}$ cell (8). The effect of pump type and flow rate for disintegrating tablets is more complex to interpret, as local fluid velocities will affect the disintegration of the tablet in addition to dissolution of the particles produced post-disintegration.

Overall, fluid velocities within the cells of FTC apparatus are markedly lower than in the paddle apparatus at $50 \mathrm{rpm}$ or basket apparatus at $100 \mathrm{rpm}$ (Figure 2). Even in the smaller $\varnothing 12 \mathrm{~mm}$ cell, the maximum simulated velocity occurring around the time of peak inflow is approximately $0.014 \mathrm{~m} / \mathrm{second}$ (at $17 \mathrm{~mL} / \mathrm{min}$ ) in comparison to approximately $0.049 \mathrm{~m} / \mathrm{second}$ at $1 \mathrm{~mm}$ from the top of a compact located in the center of the vessel base (Table 2 ) in the paddle apparatus $(30,45,53)$. However fluid velocity is not the only parameter influencing dissolution rate, and effects of media renewal through the cell must be considered when anticipating dissolution rates in the various apparatuses.

\section{CONCLUSION}

Whereas it is widely known that the hydrodynamic environment can affect the dissolution rate, hydrodynamic characteristics of dissolution conditions are not routinely monitored as part of dissolution testing, aside from setting an agitation rate and attempting to minimize variability. The current review describes how each apparatus has certain hydrodynamic characteristics. These have been explored to varying extents and determined using CFD and qualitative and quantitative flow visualization and the velocimetric methods outlined. All methods have the advantage of revealing more information about hydrodynamics in the apparatus than can be ascertained from knowledge of the agitation rate and mechanism alone. On the other hand, all methods have limitations.
Flow velocimetry methods tend to produce data that are, in some way, limited to the location and specific time of measurement, although frequent measurements can generally be taken from multiple locations. On the other hand, CFD can reveal data from any time and any location, but is limited to the accuracy of the simulation. A more accurate simulation is more computationally expensive. Therefore, hydrodynamic findings should always be interpreted in the context of the limitations of the methodology used. Notwithstanding that, the hydrodynamic features described in this review, in many cases, reveal similar findings from several studies and different methodologies. It is difficult to directly compare hydrodynamics between apparatuses in terms of the anticipated effects on dissolution, as dosage form location and behavior (i.e., motion and disintegration) during a dissolution test can also vary between apparatuses. Thus, interpretation of the hydrodynamic environment should not be over-simplified. For example, a dissolution set-up cannot be easily classified as "laminar" or "turbulent" as the hydrodynamics can vary with tablet location within the apparatus, and turbulence is a specific phenomenon in fluid dynamics. Additionally, the dosage form itself, through its geometry and solubility characteristics, can affect the hydrodynamic environment in which it is located. The multitude of studies now present in the literature and referred to in this review have provided insight into the hydrodynamics present in the paddle, basket, and flow-through apparatuses. Hydrodynamics should be considered when evaluating the design or selection of dissolution test conditions and interpreting variation in dissolution test results. However, there is scope for further hydrodynamic characterization, especially in media with different hydrodynamic properties (e.g., viscosity) and specific situations where the impact of the presence, location, and behavior of a dosage form within the dissolution test is unknown.

\section{ACKNOWLEDGEMENTS}

V Todaro and AM Healy acknowledge funding from the Synthesis and Solid State Pharmaceutical Centre (SSPC) and Science Foundation Ireland (Grant Number: 12/RC/2275).

\section{REFERENCES}

1. European Pharmacopoeia, 8th ed.; European Directorate for the Quality of Medicines \& Healthcare, Council of Europe: Strasbourg, France, 2016.

2. The United States Pharmacopeia and National Formulary USP 39-NF 34; The United States Pharmacopeial Convention, Inc.: Rockville, MD, 2016.

3. Japanese Pharmacopoeia, 17th ed.; Ministry of Health, Labour, and Welfare: Tokyo, Japan, 2016. 
4. D'Arcy, D. M.; Persoons, T. Mechanistic modelling and mechanistic monitoring: simulation and shadowgraph imaging of particulate dissolution in the flow-through apparatus. J. Pharm. Sci. 2011, 100 (3), 1102-15. DOI: 10.1002/jps.22337.

5. Sugano, K. Aqueous boundary layers related to oral absorption of a drug: from dissolution of a drug to carrier mediated transport and intestinal wall metabolism. Mol. Pharmaceutics. 2010, 7 (5), 1362-73. DOI: 10.1021/mp1001119.

6. Eaton, J.; Deng, G.; Hauck, W. W.; Brown, W.; Manning, R. G.; Wahab, S. Perturbation Study of Dissolution Apparatus Variables-A Design of Experiment Approach. Dissolution Technol. 2007, 14 (1), 20-26. DOI: 10.14227/DT140107P20.

7. Fotaki, N.; Oluwasanmi, O.; Rampal, Y.; Baxevanis, F.; Grove, G. Effect of glass bead size on drug dissolution in the flow through cell apparatus. AAPS Annual Meeting; 2014 November; San Diego, California, USA.

8. Yoshida, H.; Kuwana, A.; Shibata, H.; Izutsu, K.; Goda, Y. Effects of Pump Pulsation on Hydrodynamic Properties and Dissolution Profiles in Flow-Through Dissolution Systems (USP 4). Pharm Res. 2016, 33 (6), 1327-36. DOI: 10.1007/s11095-016-1874-8.

9. White, F. M. Fluid Mechanics. 7th ed.; McGraw Hill: New York, 2009.

10. Adrian, R. J. Particle-imaging techniques for experimental fluid mechanics. Annu. Rev. Fluid Mech. 1991, 23, 261-304. DOI: 10.1146/annurev.fl.23.010191.001401.

11. Melling, A. Tracer particles and seeding for particle image velocimetry. Meas. Sci. Technol. 1997, 8 (12), 1406. DOI: 10.1088/0957-0233/8/12/005.

12. Raffel, M.; Willert, C. E.; Wereley, S. T.; Kompenhans, J. Particle Image Velocimetry: A Practical Guide. 2nd ed.; Springer-Verlag: Berlin, 2007.

13. Bocanegra, L. M.; Morris, G. J.; Jurewicz, J. T.; Mauger, J. W. Fluid and particle laser Doppler velocity measurements and mass transfer predictions for the USP paddle method dissolution apparatus. Drug Dev. Ind. Pharm. 1990, 16 (9), 1441-64. DOI: 10.3109/03639049009074376.

14. Serrano, D. R.; Persoons, T.; D'Arcy, D. M.; Galiana, C.; DeaAyuela, M. A.; Healy, A. M. Modelling and shadowgraph imaging of cocrystal dissolution and assessment of in vitro antimicrobial activity for sulfadimidine/4-aminosalicylic acid cocrystals. Eur. J. Pharm. Sci. 2016, 89, 125-36. DOI: 10.1016/j.ejps.2016.04.030.

15. Tropea, C.; Yarin, A. L.; Foss, J. F. Springer Handbook of Experimental Fluid Mechanics; Springer-Verlag: Berlin, 2007.

16. Diebold, S. M.; Dressman, J. B. Hydrodynamik kompendialer Lösungsgeschwindigkeits-Testapparaturen Paddle und Basket. Pharm. Ind. 2001, 63 (1), 94-104.

17. Fyfe, C. A.; Grondey, H.; Blazek-Welsh, A. I.; Chopra, S. K.; Fahie, B. J. NMR imaging investigations of drug delivery devices using a flow-through USP dissolution apparatus. J. Controlled Release. 2000, 68 (1), 73-83. DOI: 10.1016/S0168-3659(00)00237-6.

18. Kulinowski, P.; Dorozynski, P.; Mlynarczyk, A.; Weglarz, W. P. Magnetic resonance imaging and image analysis for assessment of HPMC matrix tablets structural evolution in USP Apparatus 4.
Pharm. Res. 2011, 28 (5), 1065-73. DOI: 10.1007/s11095-0100357-6.

19. Nott, K. P. Magnetic resonance imaging of tablet dissolution. Eur. J. Pharm. Biopharm. 2010, 74 (1), 78-83. DOI: 10.1016/j. ejpb.2009.07.003.

20. Mauger, J.; Ballard, J.; Brockson, R.; De, S.; Gray, V.; Robinson, D. Intrinsic dissolution performance testing of the USP dissolution apparatus 2 (rotating paddle) using modified salicylic acid calibrator tablets: proof of principle. Dissolution Technol. 2003, 10, 6-15. DOI: 10.14227/DT100303P6.

21. Kukura, J.; Baxter, J. L.; Muzzio, F.J. Shear distribution and variability in the USP Apparatus 2 under turbulent conditions. Int. J. Pharm. 2004, 279 (1-2), 9-17. DOI: 10.1016/j.jpharm.2004.03.033.

22. Unger, D. R.; Muzzio, F. J. Laser-induced fluorescence technique for the quantification of mixing in impinging jets. AlChE J. 1999, 45 (12), 2477-86. DOI: 10.1002/aic.690451203.

23. Wendt, J. F. Computational Fluid Dynamics: An Introduction, 3rd ed.; Springer-Verlag: Berlin, 2009.

24. McCarthy, L. G.; Kosiol, C.; Healy, A. M.; Bradley, G.; Sexton, J. C.; Corrigan, O. I. Simulating the hydrodynamic conditions in the United States Pharmacopeia paddle dissolution apparatus. AAPS PharmSciTech. 2003, 4 (2), 83-98. DOI: 10.1208/pt040222.

25. Baxter, J. L.; Kukura, J.; Muzzio, F. J. Hydrodynamics-induced variability in the USP apparatus II dissolution test. Int. J. Pharm. 2005, 292 (1-2), 17-28. DOI: 10.1016/j.ijpharm.2004.08.003.

26. Bai, G.; Armenante, P. M.; Plank, R. V.; Gentzler, M.; Ford, K.; Harmon, P. Hydrodynamic investigation of USP dissolution test apparatus II. J. Pharm. Sci. 2007, 96 (9), 2327-49. DOI: 10.1002/ jps.20818.

27. Bai, G.; Wang, Y.; Armenante, P. M. Velocity profiles and shear strain rate variability in the USP Dissolution Testing Apparatus 2 at different impeller agitation speeds. Int. J. Pharm. 2011, 403 (1), 1-14. DOI: 10.1016/j.ijpharm.2010.09.022.

28. McCarthy, L. G.; Bradley, G.; Sexton, J. C.; Corrigan, O. I.; Healy, A. M. Computational fluid dynamics modeling of the paddle dissolution apparatus: agitation rate, mixing patterns, and fluid velocities. AAPS PharmSciTech. 2004, 5 (2), 50-9. DOI: 10.1208/ pt050231.

29. Bai, G.; Armenante, P. M. Velocity distribution and shear rate variability resulting from changes in the impeller location in the USP dissolution testing apparatus II. Pharm. Res. 2008, 25 (2), 320-36. DOI: 10.1007/s11095-007-9477-z.

30. D’Arcy, D. M.; Corrigan, O. I.; Healy, A. M. Hydrodynamic simulation (computational fluid dynamics) of asymmetrically positioned tablets in the paddle dissolution apparatus: impact on dissolution rate and variability. J. Pharm. Pharmacol. 2005, 57, 1243-50. DOI: 10.1211/jpp.57.10.0002.

31. Bai, G.; Armenante, P. M. Hydrodynamic, mass transfer, and dissolution effects induced by tablet location during dissolution testing. J. Pharm. Sci. 2009, 98 (4), 1511-31. DOI: 10.1002/ jps.21512.

32. Higuchi, M.; Yoshihashi, Y.; Tarada, K.; Sugano, K. Minimum rotation speed to prevent coning phenomena in compendium 
paddle dissolution apparatus. Eur. J. Pharm. Sci. 2014, 65, 74-8. DOI: 10.1016/j.ejps.2014.09.010.

33. Higuchi, M; Nishida, S.; Yoshihashi, Y.; Tarada, K.; Sugano, K. Prediction of coning phenomena for irregular particles in paddle dissolution test. Eur. J. Pharm. Sci. 2015, 76, 213-6. DOI: 10.1016/j.ejps.2015.05.019.

34. Ameur, H.; Bouzit, M. 3D hydrodynamics and shear rates' variability in the United States Pharmacopeia Paddle Dissolution Apparatus. Int. J. Pharm. 2013, 452 (1-2), 42-51. DOI: 10.1016/j. ijpharm.2013.04.049.

35. Baxter, J. L.; Kukura, J.; Muzzio, F. J. Shear-induced variability in the United States Pharmacopeia Apparatus 2: modifications to the existing system. AAPS J. 2006, 7 (4), E857-64. DOI: 10.1208/ aapsj070483.

36. Wang, Y.; Armenante, P. M. A novel off-center paddle impeller (OPI) dissolution testing system for reproducible dissolution testing of solid dosage forms. J. Pharm. Sci. 2012, 101 (2), 74660. DOI: 10.1002/jps.22783.

37. Mirza, T.; Joshi, Y.; Liu, Q.; Vivilecchia, R. Evaluation of Dissolution Hydrodynamics in the USP, Peak ${ }^{\mathrm{TM}}$ and Flat-Bottom Vessels Using Different Solubility Drugs. Dissolution Technol. 2005, 1 (1), 11-6. DOI: 10.14227/DT120105P11.

38. Zhang, Y.; Bredael, G.; Armenante, P. M. Dissolution of prednisone tablets in the presence of an arch-shaped fiber optic probe in a USP dissolution testing apparatus 2. J. Pharm. Sci. 2013, 102 (8), 2718-29. DOI: 10.1002/jps.23651.

39. Klein, S. The Mini Paddle Apparatus-a Useful Tool in the Early Developmental Stage? Experiences with Immediate-Release Dosage Forms. Dissolution Technol. 2006, 13 (4), 6-11. DOI: 10.14227/DT130406P6.

40. Klein, S.; Shah, V. P. A standardized mini paddle apparatus as an alternative to the standard paddle. AAPS PharmSciTech. 2008, 9 (4), 1179-84. DOI: 10.1208/s12249-008-9161-6.

41. Scheubel, E.; Lindenberg. M.; Beyssac, E.; Cardot, J-M. Small Volume Dissolution Testing as a Powerful Method during Pharmaceutical Development. Pharmaceutics. 2010, 2 (4), 35163. DOI: $10.3390 /$ pharmaceutics2040351.

42. Stamatopoulos, K.; Batchelor, H. K.; Alberini, F.; Ramsay, J.; Simmons, M. J. Understanding the impact of media viscosity on dissolution of a highly water soluble drug within a USP 2 mini vessel dissolution apparatus using an optical planar induced fluorescence (PLIF) method. Int. J. Pharm. 2015, 495 (1), 362-73. DOI: 10.1016/j.ijpharm.2015.09.002.

43. Locher, K.; Borghardt, J. M.; Frank, K. J.; Kloft, C.; Wagner, K. G. Evolution of a mini-scale biphasic dissolution model: Impact of model parameters on partitioning of dissolved API and modelling of in vivo-relevant kinetics. Eur. J. Pharm. Biopharm. 2016, 105, 166-75. DOI: 10.1016/j.ejpb.2016.06.008.

44. D'Arcy, D. M.; Corrigan, O. I.; Healy, A. M. Evaluation of hydrodynamics in the basket dissolution apparatus using computational fluid dynamics--dissolution rate implications. Eur. J. Pharm. Sci. 2006, 27 (2-3), 259-67. DOI: 10.1016/j. ejps.2005.10.007.
45. D'Arcy, D. M. Use of computational fluid dynamics to investigate the relationship between hydrodynamics and rates of dissolution; Dublin: Trinity College Dublin, 2007.

46. Morihara, M.; Aoyagi, N.; Kaniwa, N.; Katori, N.; Kojim, S. Hydrodynamic flows around tablets in different pharmacopeial dissolution tests. Drug Dev. Ind. Pharm. 2002, 28 (6), 655-62. DOI: 10.1081/DDC-120003856.

47. Fotaki, N. Flow-Through Cell Apparatus (USP Apparatus 4): Operation and Features. Dissolution Technol. 2011, 18 (4), 46-9. DOI: 10.14227/DT180411P46.

48. Banakar UV. Pharmaceutical Dissolution Testing; New York: Marcel Dekker; 1992.

49. D’Arcy, D. M.; Liu, B.; Persoons, T.; Corrigan, O. I. Hydrodynamic Complexity Induced by the Pulsing Flow Field in USP Dissolution Apparatus 4. Dissolution Technol. 2011, 18 (4), 6-13. DOI: 10.14227/DT180411P6.

50. Eaton, J. W.; Tran, D.; Hauck, W. W.; Stippler, E. S. Development of a performance verification test for USP apparatus 4. Pharm. Res. 2012, 29 (2), 345-51. DOI: 10.1007/s11095-011-0559-6.

51. Kakhi, M. Classification of the flow regimes in the flow-through cell. Eur. J. Pharm. Sci. 2009, 37 (5), 531-44. DOI: 10.1016/j. ejps.2009.04.003.

52. Kakhi, M. Mathematical modeling of the fluid dynamics in the flow-through cell. Int. J. Pharm. 2009, 376 (1-2), 22-40. DOI: 10.1016/j.jpharm.2009.04.012.

53. D'Arcy, D. M.; Liu, B.; Bradley, G.; Healy, A. M.; Corrigan, O. I. Hydrodynamic and species transfer simulations in the USP 4 dissolution apparatus: considerations for dissolution in a low velocity pulsing flow. Pharm. Res. 2010, 27 (2), 246-58. DOI: 10.1007/s11095-009-0010-4.

54. D'Arcy, D. M.; Liu, B.; Corrigan, O. I. Investigating the effect of solubility and density gradients on local hydrodynamics and drug dissolution in the USP 4 dissolution apparatus. Int. J. Pharm. 2011, 419 (1-2), 175-85. DOI: 10.1016/j.jpharm.2011.07.048.

55. Stevens, L. E.; Missel, P. J. Impact of density gradients on flowthrough dissolution in a cylindrical flow cell. Pharm. Dev. Technol. 2006, 11 (4), 529-34. DOI: 10.1080/10837450600970441.

56. Shiko, G.; Gladden, L. F.; Sederman, A. J.; Connolly, P. C.; Butler, J. M. MRI studies of the hydrodynamics in a USP 4 dissolution testing cell. J. Pharm. Sci. 2011, 100 (3), 976-91. DOI: 10.1002/ jps.22343.

57. Cammarn, S. R.; Sakr, A. Predicting dissolution via hydrodynamics: salicylic acid tablets in flow through cell dissolution. Int. J. Pharm. 2000, 201 (2), 199-209. DOI: 10.1016/S0378-5173(00)00415-4.

58. Shiko, G.; Sederman, A. J.; Gladden, L. F. MRI technique for the snapshot imaging of quantitative velocity maps using RARE. J. Magn. Reson. 2012, 216, 183-91. DOI: 10.1016/j. jmr.2012.01.021.

59. Yoshida, H.; Kuwana, A.; Shibata, H.; Izutsu, K.; Goda, Y. Particle Image Velocimetry Evaluation of Fluid Flow Profiles in USP 4 FlowThrough Dissolution Cells. Pharm. Res. 2015, 32 (9), 2950-9. DOI: 10.1007/s11095-015-1676-4. 